Cochrane erstellt systematische Übersichtsarbeiten zu medizinischen Forschungsfragen und schafft damit eine zuverlässige Grundlage für die evidenzbasierte Medizin. Seit 2016 haben alle in der Schweiz wohnhaften Personen kostenlosen Zugang zur Cochrane Library. Im Rahmen einer vierteiligen Artikelserie stellen wir die Cochrane Library vor. In diesem Artikel berichtet Hausarzt Dr. med. Bruno Kissling von seinen Erfahrungen im Umgang mit der Cochrane Library.

Dr. med. Christoph Bosshard Vizepräsident der FMH, Departementsverantwortlicher DDQ / SAQM

\title{
Cochrane Library und Hausarztmedizin
}

\section{Bruno Kissling}

Dr. med., Hausarzt im Ruhestand

\section{Die Cochrane Library ist vielen Hausärzten nicht geläufig}

Meine Umfrage unter Kolleginnen und Kollegen hat ergeben, dass es ihnen ähnlich ergeht wie mir: Die meisten haben vom Angebot des Gratiszugangs zur Cochrane Library gehört, haben jedoch bisher kaum Gebrauch von ihr gemacht. Sie nutzen oftmals andere Informationsquellen, die besser auf ihre Bedürfnisse abgestimmt sind.

\section{Das Wesen der Hausarztmedizin ${ }^{1}$ und ihre Anforderung an Entscheidungshilfen}

1 The European Definition of General Practice / Family Medicine 2002/ 2011 Wonca Europe www.woncaeurope.org/ gp-definitions

2 Family medicine ICPC Research using Electronic medical records - ein vorerst zeitlich unlimitiertes Forschungsprojekt unter Leitung des Instituts für Hausarztmedizin der Universität Zürich ( $\mathrm{IH}$ AMZ

3 Andreas Sönnichsen, Univ.-Prof., Zentrum für Public Health, Medizi nische Universität Wien, Abteilung für Allgemein und Familienmedizin, erster Vorsitzender im geschäftsführenden Vorstand des Deutschen Netzwerks Evidenzbasierte Medizin (DNEbM), Herausgeber von IWIMED, essence of medicine, mit hausarztspezifischen Guidelines für Ärzte und Patienten, Mitherausgeber EbM-Guidelines: Evidenzbasierte Medizin für Praxis \& Klinik.
In der Hausarztpraxis behandeln wir täglich eine grosse Zahl von Patientinnen und Patienten in relativ kurzen Konsultationen. Gemäss einer internen Erhebung von FIRE $^{2}$ besprechen Hausärzte pro Konsultation 2,7 Probleme. Eine hohe Zahl unserer Patientinnen sind chronisch krank oder multimorbid, oft gebrechlich. Viele Symptome sind durch den psychosozialen Kontext des Patienten und weitere gleichzeitig vorliegende Krankheitszustände mitgeprägt. Das individuelle Krankheitserleben der Patienten ist für die personen- und kontextbezogenen hausärztlichen Entscheidungen genauso relevant wie die statistische Signifikanz eines Unterschieds zwischen zwei Behandlungsalternativen. Die Summe dieser Begebenheiten steigert die Ungewissheit oder Unsicherheit betreffend das Symptom oder das Problem sowie die personenund kontextbezogenen Entscheidungen von Arzt und Patient. Aus dieser Komplexität resultieren besondere Anforderungen an wissenschaftliche Entscheidungshilfen für Hausärzte.

\section{Cochrane Library in der Hausarztmedizin}

Kann die Cochrane Library bei diesen hausarztmedizinischen Besonderheiten den Arzt und Patienten bei

\section{Zusammenfassung}

Die Cochrane Library dürfte weltweit allen Ärztinnen und Ärzten bekannt sein. Im Internet erreicht man die Website in Sekundenschnelle. Seit 2016 haben alle Bewohnerinnen und Bewohner der Schweiz, als Angebot der Schweizerischen Akademie der Medizinischen Wissenschaften, des Bundesamts für Gesundheit und der Schweizer Universitätsbibliotheken, gratis Zugang zu dieser medizinischen Datenbank. Im Rahmen dieses Artikels gehe ich folgenden Fragen nach: Kennen Hausärztinnen und Hausärzte die Cochrane Library und nutzen sie diese? Und kann ein Hausarzt in den Cochrane Reviews Antworten auf seine meist komplexen hausarztspezifischen Fragestellungen finden?

ihrer gemeinsamen Entscheidungsfindung unterstützen?

Professor Andreas Sönnichsen ${ }^{3}$, Hausarzt und Wissenschaftler mit besonderem Interesse an der evidenzbasierten Medizin, schreibt auf meine Mail-Anfrage sinngemäss: "Als Point-of-Care Tool ist die Cochrane Library für praktisch tätige Hausärztinnen und Hausärzte ungeeignet. Selbst wenn sie im Umgang mit ihr geübt sind, können sie nicht während der Sprechstunde in Cochrane suchen. Sie wäre eher etwas für die eigene Fortbildung oder für spezielle Fragestellungen, die sie am Abend oder Wochenende recherchieren können. Der Cochrane Review fokussiert in der Regel auf eine einzige, ganz spezifische Fragestellung. Viele, gerade hausärztliche Fragestellungen sind in Cochrane Reviews jedoch nicht abgebildet. Dies aber nicht, weil man die Hausärzte 'vergessen' hat, sondern, weil es generell wenig Studien zu spezifischen hausärztlichen Fragestellungen gibt.»

Daraus lässt sich schliessen: Hausärztliche Point-ofCare-Entscheidungshilfen können nur bei ganz eng fokussierten Fragestellungen direkt aus Recherchen in der Cochrane Library gewonnen werden. 


\section{Recherche für eine komplexe Fragestellung}

Für das Verfassen dieses Artikels taste ich mich an die Cochrane Library heran. Allmählich entdecke ich die vielfältige Struktur und die Suchmöglichkeiten dieser elektronischen Datenbank mit ihren Tausenden von Reviews und Zehntausenden von Trials ${ }^{4}$.

Ich versuche eine Antwort zu finden für die Behandlung meiner Patientin mit einer Hyperthyreose mit erhöhten TSH-Rezeptor-Antikörpern entsprechend einem Morbus Basedow. Da meine Patientin gleichzeitig eine klinisch mässig manifeste knotige Struma hat, möchten wir wissen, ob eine Strumektomie eine valable Therapie wäre, um beide Probleme zusammen zu lösen.

Im Suchfeld auf der Hauptseite der Cochrane Library gebe ich Morbus Basedow ein und grenze die Suche nach Title Abstract Keyword ein. Es erscheint ein Review "Intraoperative neuromonitoring versus visual nerve identification for prevention of recurrent laryngeal nerve injury in adults undergoing thyroid surgery». Wenn ich unter All Text suche, erscheinen vier Trials, darunter «Drug treatment of immune hyperthyroidism (Basedow disease). Patient selection, longterm follow-up and prevention of recurrence» und «Basedow's disease - hemithyroidectomy and contralateral near-total strumectomy in comparison with bilateral near-total strumectomy". Darunter sind keine Resultate, die meine Fragestellung vollumfänglich beantworten. Unter Eingabe von goiter und Suche unter All Text erscheinen 31 Cochrane Reviews. Drei Reviews nehmen meine Frage am Rand auf: «Total or near-total thyroidectomy versus subtotal thyroidectomy for multinodular non-toxic goitre in adults", "Antithyroid drug regimen for treating Graves' hyperthyroidism» sowie "Radioiodine therapy versus antithyroid medications for Graves' disease». Ich führe meine Suche unter Advanced Search und den Suchbegriffen "Basedow disease" kombiniert mit "drug therapy" und "surgery" r therapyn und usurgeryn

\section{Über Cochrane}

Cochrane ist ein globales, unabhängiges Netzwerk von Wissenschaftlerinnen und Wissenschaftlern, Gesundheitsfachleuten, Patienten und Patientinnen sowie anderen Personen mit gesundheitsbezogenen Interessen. Zur Arbeit von Cochrane tragen ca.11000 Mitglieder und über 68000 Unterstützer aus über 130 Ländern bei. Auf wissenschaftliche Methoden gestützt, stellen sie Gesundheitsinformationen bereit, die zuverlässig und frei von kommerziellen Sponsorengeldern sind. Jeder Cochrane Review widmet sich einer klar formulierten Fragestellung. Zu deren Beantwortung sucht ein Autorenteam alle vorhandenen Originalstudien, welche die zuvor definierten Einschlusskriterien erfüllen. Anschliessend werden die eingeschlossenen Studien bewertet, um zu bestimmen, ob es zuverlässige Evidenz zu einer bestimmten Behandlung, Diagnostik oder vorbeugenden Massnahme gibt. Wenn möglich, werden die Einzelergebnisse in einer Metaanalyse kombiniert. Cochrane Reviews werden vor der Veröffentlichung im Peer-Review-Verfahren von Fachexperten begutachtet. Über 8000 Reviews sind bisher auf www.cochranelibrary.com zugänglich. Neben dem oft sehr ausführlichen Volltext stehen verschiedene Kurzformate zur Verfügung. Für die klinische Praxis besonders relevante Reviews werden unter Clinical Answers in einem Frage-Antwort-Format dargestellt. weiter und stosse unter insgesamt sieben Reviews, neben bereits vorher gefundenen Reviews, zusätzlich auf «Thyroid surgery for Graves' disease and Graves' ophthalmopathy». Unter Clinical Answers werden die Ergebnisse von drei dieser Reviews kompakt wiedergegeben.

\section{Erkenntnisse als Basis für die Patientenberatung}

Vier Reviews sowie die Clinical Answers kann ich für die Beantwortung meiner Fragestellung beiziehen. Nach intensiver Suche gelange ich zu folgenden Eckpunkten für die Besprechung mit meiner Patientin: Eine totale Thyreoidektomie, die besser sei als eine subtotale Thyreoidektomie, könnte zur gleichzeitigen Behandlung des Morbus Basedow und der Struma in Betracht gezogen werden. Diese erfordert jedoch eine lebenslange Substitutionstherapie mit Schilddrüsenhormonen und ist zudem mit einem erhöhten Risiko einer lebenslangen Kalzium-Stoffwechselstörung behaftet. Beide Operationsmethoden haben keinen unterschiedlichen Einfluss auf die Rückbildung einer Ophthalmopathie und betreffend das Risiko einer Rekurrensparese. Der Morbus Basedow wird mit einer medikamentösen Therapie (Methimazol) während 12 bis 18 Monaten behandelt; wegen weniger Nebenwirkung in ein- und austitrierender Weise. Eine anschliessende Substitutionsbehandlung mit Schilddrüsenhormonen schützt nicht vor einem Rezidiv. Eine Radiojod-Therapie bringt keinen Vorteil gegenüber Methimazol (Propylthiouracil wurde nicht getestet), erhöht aber das Risiko für eine Ophthalmopathie. Aus meiner hausärztlichen Sicht werde ich meiner Patientin folgendes Vorgehen als Ausgangslage zur gemeinsamen Entscheidung vorschlagen: In Anbetracht der Datenlage dürfte es sinnvoll und sicherer sein, zuerst den Morbus Basedow medikamentös zu behandeln. Die mässig störende Struma würde besser in einem zweiten Schritt, nach Abheilung des Morbus Basedow, chirurgisch behandelt - vorzugsweise mit einer weniger riskanten partiellen Thyreoidektomie, auch wenn diese als einzigen Nachteil gegenüber einer totalen Thyreoidektomie ein etwas erhöhtes Risiko für ein Struma-Rezidiv mit sich bringt.

\section{Fazit}

Die Cochrane Library kennenzulernen lohnt sich. Es ist spannend, auf dieser eindrücklichen Riesendatenbank $\mathrm{zu}$ flanieren. Der Zeitaufwand ist anfänglich gross, doch mit mehr Übung geht es immer leichter. Das Finden von Antworten auf eine komplexe Fragestellung in der Hausarztmedizin ist herausfordernd, für eng gefasste Fragestellungen jedoch möglich. 